\title{
Biomarcadores y endotipos en la sepsis. Nuevas evidencias
}

\section{Biomarkers and sepsis endotypes. New evidence}

\author{
Ansony R. Godínez-Vidal' ${ }^{*}$, Raúl Carrillo-Esper ${ }^{2}$ y Ricardo Cabello-Aguilera ${ }^{3}$ \\ ${ }^{1}$ Servicio de Cirugía General, Hospital HMG Coyoacán; ${ }^{2}$ Servicio de Terapia Intensiva, Hospital HMG Coyoacán; ${ }^{3}$ Servicio de Terapia Intensiva, \\ Instituto Nacional de Rehabilitación "Luis Guillermo Ibarra Ibarra". Ciudad de México, México
}

\section{Resumen}

Antecedentes: La sepsis es una causa importante de enfermedad y muerte en todo el mundo, y es un proceso complejo y heterogéneo. Ahora se reconoce que los marcadores biológicos mejoran la clasificación de la sepsis y pueden facilitar la identificación de distintas subclases o endotipos de pacientes. Objetivo: Analizar los conceptos actuales en biología traslacional que ayudan a entender la heterogeneidad en la respuesta del paciente con sepsis. Conclusiones: Hay una serie de biomarcadores que podrían ser útiles para construir endotipos compuestos que predicen resultados no complicados en la sepsis. Estos endotipos podrían ayudar a identificar a los pacientes que merecen el inicio temprano de antimicrobianos o la interrupción de estos.

Palabras clave: Sepsis. Biología. Endotipos. Biomarcadores.

\section{Abstract}

Background: Sepsis is an important cause of disease and death worldwide, and it has a complex and heterogeneous disease process. It is now recognized that biological markers improve sepsis classification and may facilitate the identification of different subclasses or endotypes of patients. Objective: To analyze the current concepts in translational biology that help to understand the heterogeneity in the response of the patient with sepsis. Conclusions: There are a number of biomarkers that could be useful for constructing endo-compound types that predict uncomplicated results in sepsis. These endotypes could help identify patients who deserve early antimicrobial onset or discontinuation.

Key words: Sepsis. Biology. Endotypes. Biomarkers.

\section{Introducción}

La sepsis es una causa importante de enfermedad y muerte en todo el mundo, y es un proceso complejo y heterogéneo que está asociado o causado por una variedad de microorganismos y fuentes de infección.
Actualmente hay un cambio prometedor de la predicción del resultado a una comprensión de la diversidad en la respuesta del huésped a la sepsis basada en la patobiología. Ahora se reconoce que los marcadores biológicos mejoran la clasificación de la sepsis y pueden facilitar la identificación de distintas subclases o endotipos de pacientes.

\section{Correspondencia:}

*Ansony R. Godínez-Vidal

Árbol del Fuego 80

Col. El Rosario, Del. Coyoacán 
El objetivo de este trabajo es hacer una revision de los conceptos actuales en biología traslacional que ayudan a entender la heterogeneidad en la respuesta de los pacientes con sepsis.

\section{Perfiles de la sepsis}

Uno de los primeros estudios en utilizar el agrupamiento jerárquico para estudiar los subgrupos de sepsis dentro de la población de la unidad de cuidados intensivos ( $\mathrm{UCl}$ ) se realizó en una cohorte de 98 niños ingresados con diagnóstico de shock séptico'.

Mediante patrones de expresión del genoma se identificaron tres subclases: endotipo A (29\%), endotipo B $(46 \%)$ y endotipo C (26\%). Estas tres clases diferían significativamente en los fenotipos clínicos, incluida la mortalidad en la UCI (la más alta en el endotipo $A$, con un $36 \%$, frente al $11 \%$ y el $12 \%$ para los endotipos B y C, respectivamente), la gravedad de la enfermedad y el grado de insuficiencia orgánica (ambos más altos en el endotipo A), y la edad (más joven en el endotipo A).

Una metodología similar se ha aplicado recientemente a la población adulta en estado crítico. Entre 265 pacientes con diagnóstico de sepsis debida a neumonía adquirida en la comunidad, como parte del estudio Genomic Advances in Sepsis (GAinS), los perfiles transcriptómicos definieron dos señales de respuesta a la sepsis: SRS1 (41\%) y SRS2 $(59 \%)^{2}$. En relación con los pacientes SRS2, los pacientes del grupo SRS1 tuvieron una mortalidad mayor de 14 días (22\% frente a 10\%). La asignación de SRS1 se asoció con inmunosupresión relativa, tolerancia a endotoxinas, agotamiento de células $\mathrm{T}$, regulación a la baja del antígeno leucocitario humano (HLA) de clase II y trastornos metabólicos (cambio de fosforilación oxidativa a glucólisis). De los más de 3000 genes expresados diferencialmente, siete predijeron de manera confiable la pertenencia al SRS. En futuros estudios, los pacientes asignados prospectivamente a SRS1 pueden beneficiarse de las terapias que estimulan su sistema inmunitario y previenen la infección nosocomial. Este enfoque analítico fue utilizado posteriormente por los mismos investigadores para estudiar los patrones de expresión génica en 117 pacientes con peritonitis fe$\mathrm{Cal}^{3}$. Se identificaron de nuevo dos grupos distintos, SRS1_FP (46\%) y SRS2_FP (54\%), y los pacientes en el grupo SRS1_FP también tuvieron una mortalidad mayor de 14 días (19\% frente a 4\%). Los hallazgos se correlacionaron fuertemente con los grupos de SRS identificados en el estudio $\mathrm{CAP}^{2}$, mostrando nuevamente el aumento de la tolerancia a la endotoxina y la activación de las células $T$, pero también la muerte celular, la apoptosis y la necrosis. De los más de 1000 genes expresados diferencialmente se derivó un conjunto más simple de seis genes que predijo la pertenencia al grupo. Cabe destacar que cuando los patrones de expresión génetica que distinguieron a los grupos de SRS se probaron en la cohorte pediátrica antes descrita ${ }^{1}$ no se observó el mismo aumento (es decir, SRS1_FP ${ }^{3}$ y el endotipo $A^{1}$ ).

Una mejor comprensión de la variación genética humana y el uso de la metabolómica son aspectos clave para separar grupos de pacientes con respuestas similares, y el uso de estas técnicas podría ayudar a estratificar el riesgo de los pacientes. Scicluna, et al. ${ }^{4}$ informan los resultados de un estudio de transcriptómica basado en una matriz que utiliza muestras de sangre recogidas al ingreso en la $\mathrm{UCl}$ de 526 pacientes con sepsis de dos $\mathrm{UCl}$ en los Países Bajos (cohorte de descubrimiento y primera validación), y en 265 pacientes con sepsis asociada a neumonía de 29 $\mathrm{UCl}$ en el Reino Unido (segunda cohorte de validación), con el objetivo de definir distintos subtipos funcionales y homogéneos, es decir, endotipos. Los transcriptomos sanguíneos de estos endotipos tuvieron una respuesta distinta del huésped, que fueron desde la inmunosupresión hasta la hiperinflamación. Encontraron que podría haber cuatro endotipos, que son los grupos en los cuales los dividen de acuerdo con su respuesta inmunitaria dominada por la genética. Cada grupo reacciona de una forma, pudiendo predecir la posibilidad de muerte en cada grupo. El grupo 1 (llamado MARS 1) es el que tiene más mortalidad y el que presenta una respuesta disminuida del patrón de reconocimiento y la señalización de citocinas, mientras que el grupo MARS 3 es el que tiene mejor pronóstico (Fig. 1). Se sabe que los altos niveles de mediadores proinflamatorios circulantes predicen un aumento de la mortalidad ${ }^{5}$, pero la mala evolucion de los pacientes también se asocia con mayores concentraciones de citocinas antiinflamatorias ${ }^{6}$. Esta observación podría reflejar la supresión de la actividad inmunitaria (comúnmente conocida como inmunoparálisis) en los pacientes con el endotipo Mars 1, lo que contribuiría al desarrollo de infecciones oportunistas que aumentan la gravedad de la sepsis.

En un grupo de 306 pacientes ingresados en la $\mathrm{UCl}$ en los Países Bajos, como parte del proyecto de diagnóstico molecular y estratificación del riesgo de sepsis (MARS), se identificaron cuatro endotipos moleculares (Mars 1-4)4. La mortalidad a los 28 días 


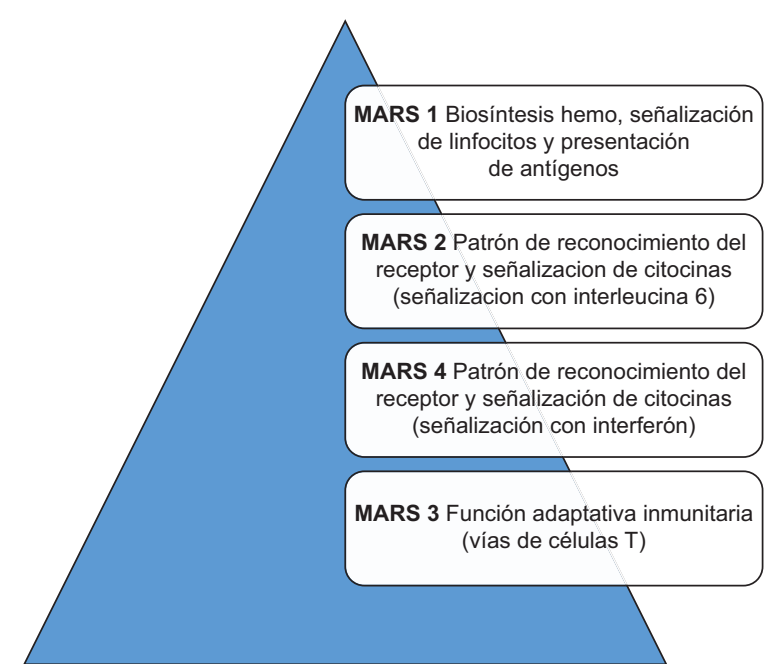

Figura 1. Endotipos de la sepsis. El grupo 1 (Ilamado MARS 1) es el que tiene más mortalidad.

difirió entre los subgrupos y fue más alta en el grupo Mars 1, con $39 \%$, en comparación con el $22 \%$ en Mars 2, el $23 \%$ en Mars 3 y el $33 \%$ en Mars 4 . El endotipo Mars 1 de mal pronóstico tuvo una disminución en la expresión de genes involucrados en funciones inmunitarias innatas y adaptativas (receptor Toll-like, señalización del factor nuclear kappa B [NF-kB], presentación de antígeno y señalización del receptor de células T) y un aumento en la expresión de las vías metabólicas celulares (biosíntesis de hemo), procesos que son análogos al agotamiento inmunitario. Los endotipos Mars 2 y 4 tenían una regulación al alza del reconocimiento de patrones y vías de citocinas (interleucina [IL] 6, NF-kB, señalización de interferón, óxido nítrico sintasa inducible), que representa un estado hiperinflamatorio. Finalmente, Mars 3 era un endotipo de bajo riesgo con expresión aumentada de vías inmunitarias adaptativas (células T ayudantes, células asesinas naturales, señalización de IL-4, desarrollo de células B), que estaba altamente correlacionado con el endotipo SRS2 de bajo ries$g^{2}$. Se obtuvo una relación de expresión de dos genes para permitir la clasificación de cada endotipo en el momento de la admisión en la UCl.

En el intento más reciente y completo de identificar subtipos de sepsis, los resultados de 14 conjuntos de datos transcriptómicos de 700 pacientes revelaron tres grupos robustos de respuesta del huésped en todo el espectro de sepsis?. Estos se denominaron: 1) inflamatorio (aumento de la señal inmunitaria adaptativa innata y reducida marcada por una mayor expresión del receptor de IL-1, actividad del receptor de reconocimiento de patrones y activación del complemento); 2) adaptativo (señal inmunitaria reducida y alta adaptativa con menor mortalidad, marcada por la señalización de interferón); y 3) coagulopatía (irregularidades en los sistemas de coagulación y del complemento, incluida la desgranulación de plaquetas y la unión de glucosaminoglicanos). Al igual que en los análisis anteriores, los tres grupos diferían en la mortalidad a 30 días, con la tasa más alta en el grupo inflamatorio, del 30\%, en comparación con el $8 \%$ en los grupos adaptativos y el $25 \%$ en los grupos con coagulopatía. Se obtuvo un clasificador simplificado de 33 genes para facilitar la asignación de grupos. La asignación al grupo inflamatorio de alta mortalidad correspondió a SRS1 y al grupo adaptativo de baja mortalidad a SRS2 ${ }^{2}$. Los cambios transcripcionales tempranos también pueden haber identificado pacientes con riesgo de complicaciones asociadas a sepsis al momento de la admisión en la UCl. Un estudio de nuestro grupo de investigación que incluyó 57 pacientes con sepsis encontró que la expresión diferencial de mediadores clave de la respuesta inicial de neutrófilos a la infección identificó a pacientes con síndrome de dificultad respiratoria aguda (SDRA) ( $n=$ 29), en comparación con aquellos con sepsis que no tenían SDRA ( $n=28)$; hallazgo que no se pudo atribuir al recuento de neutrófilos ${ }^{8}$.

Hasta la fecha, los estudios de expresión de todo el genoma en sepsis han utilizado poblaciones enteras de leucocitos. Sin embargo, hay distintos patrones de expresión génica entre subconjuntos de granulocitos y linfocitos que representan la función especializada de cada una de estas células inmunitarias ${ }^{9}$. Dado que el perfil del transcriptoma depende del tipo de célula inflamatoria, es posible que los patrones de expresión génica que distinguen las subclases reflejen diferentes poblaciones de leucocitos en lugar de las diferencias dentro de las células en la expresión génica. Estos hallazgos también requieren validación en grandes cohortes que abarquen diferentes países, ya que la variación en el origen étnico es un fuerte determinante de la expresión génica ${ }^{10}$. Sin embargo, estos estudios proporcionan evidencia de distintas categorías de la respuesta del huésped a la sepsis, y posibles objetivos terapéuticos novedosos basados en vías moleculares que distinguen los endotipos de los pacientes.

\section{Proteínas}

Un método más establecido y más factible de subclasificación biológica de los pacientes con sepsis es 
la cuantificación de las proteínas plasmáticas, y un gran número de estudios han clasificado la sepsis con este enfoque ${ }^{11-13}$. Un método notable que podría ofrecer una forma novedosa de derivar subclases de sepsis es combinar biomarcadores moleculares y de proteínas para predecir el resultado en pacientes con shock séptico. Este enfoque se utilizó para estratificar el riesgo de shock séptico pediátrico con una puntuación de riesgo previamente validada que consta de cinco biomarcadores de proteínas plasmáticas (árbol de decisión PERSEVERE) ${ }^{13}$ y la combinación de estos con cuatro evaluaciones de mortalidad de genes principales ${ }^{14}$. Se observó una mejora en el rendimiento de la puntuación de riesgo que estima el riesgo de mortalidad a 28 días (PERSEVERE-XP, el área bajo la curva aumenta de 0.78 a 0.91). Los biomarcadores plasmáticos se asociaron con inflamación disfuncional y daño celular, mientras que los genes se relacionaron con la proteína tumoral 53 (TP53, p53), un factor transcripcional que funciona como supresor tumoral, evitando la generación y la persistencia de células con daño genómico ${ }^{14}$.

\section{Metabolómica}

La metabolómica es un método en desarrollo para descifrar la heterogeneidad en la sepsis. Se refiere a la evaluación global de pequeños metabolitos en cualquier muestra biológica, que representa la expresión génica, la actividad enzimática y el paisaje fisiológico ${ }^{15}$. Se pueden detectar más de 5000 metabolitos en células, tejidos o biofluidos (componentes sanguíneos, orina) utilizando espectroscopía de resonancia magnética (RM) o espectrometría de masas; esta última es más sensible y puede detectar metabolitos poco abundantes ${ }^{16}$. La espectroscopía RM de $1 \mathrm{H}$ se utilizó para analizar y comparar muestras de suero de adultos con shock séptico y de controles en la $\mathrm{UCl}^{17}$. Se reconocieron 60 metabolitos, 31 de los cuales pudieron distinguir a pacientes con shock séptico en la $\mathrm{UCI}$, proponiendo un patrón compuesto de biomarcadores que podría diferenciar entre estos grupos de pacientes. Los metabolitos involucrados en el metabolismo energético incluían glucosa, 3-hidroxibutirato, O-acetilcarnitina, succinato, creatina, fosfato de creatina y un nivel disminuido de aminoácidos de cadena ramificada y arginina. Estos resultados sugieren que, en la sepsis temprana, los metabolitos involucrados en el metabolismo energético tienen un papel en la fisiopatología de la sepsis. La alteración de los metabolitos implicados en el metabolismo energético también se ha reconocido como clave para distinguir a los sobrevivientes de sepsis de los no sobrevivientes utilizando espectroscopía de masas para caracterizar los metabolitos de menor concentración en plasma. En un subestudio del ensayo ALBIOS (Albumin Italian Outcome Result Sepsis), que inscribió a 1818 pacientes con sepsis grave o shock séptico, se estudiaron las muestras de plasma de los días 1 y 7 de 20 pacientes, el $45 \%$ de los cuales fallecieron al día $28^{18}$. Otra gran cohorte que utilizó espectrometría de masas para estudiar los biomarcadores de metabolitos en 60 sobrevivientes de la $\mathrm{UCl}$ y 30 no sobrevivientes de la $\mathrm{UCl}$ descubrió que, de los 187 metabolitos probados, 57 estaban asociados con la mortalidad a los 28 días $^{19}$ y 31 de ellos fueron replicados en la cohorte de validación CAPSOD ${ }^{20}$. Estos metabolitos incluyeron diversos productos de lípidos, hidratos de carbono, aminoácidos y nucleótidos. Valores más altos de productos del catabolismo de tirosina y fenilalanina y valores más bajos de metabolitos lipídicos se asociaron con la mortalidad. El estudio más grande para investigar los metabolitos mediante espectrometría de masas en pacientes con sepsis incluyó 406 sujetos, de los cuales 268 se incluyeron en una cohorte de descubrimiento (42 pacientes con síndrome de respuesta inflamatoria sistémica, 67 con neumonía adquirida en la comunidad, 60 con infección intraabdominal, 73 con infección del tracto urinario y 26 con infección del torrente sanguíneo) ${ }^{21}$. El análisis también indicó una gran heterogeneidad en los patrones de metabolitos, dependiendo de la fuente de infección anatómica, y por lo tanto, se propuso un modelo de un metabolito para pronosticar un resultado desfavorable para cada tipo de infección.

\section{Biomarcadores de daño microcirculatorio}

La adrenomedulina es un péptido producido por diversos tejidos durante el estrés fisiológico e infeccioso, con diferentes funciones. Los niveles del fragmento medio regional de proadrenomedulina (MR-proADM) se correlacionan con el grado de insuficiencia orgánica en la sepsis. Elke, et al. ${ }^{22}$ demostraron que las bajas concentraciones de MR-proADM identifican pacientes con sepsis con un riesgo muy bajo de muerte durante la estadía en la UCI. EI MRproADM tiene un valor predictivo negativo (VPN) del $94 \%$ para descartar la mortalidad a los 28 días y del $96 \%$ en el día $10^{22}$. 


\section{Biomarcadores inmunitarios}

El antígeno leucocitario humano DR (HLA-DR) representa la capacidad de los monocitos para la presentación del antígeno y la conexion con las células T auxiliares, lo que permite la activación del sistema inmunitario adaptativo. EI HLA-DR en monocitos se considera un marcador sustituto de la inmunosupresión inducida por la sepsis. Los sobrevivientes muestran un aumento significativo en el porcentaje de monocitos HLA-DR positivos durante el curso de la sepsis $^{23}$. Usando citometría de flujo en un grupo de pacientes con sepsis grave, Wu, et al. ${ }^{24}$ demostraron que un incremento del $4.8 \%$ en el día 3 del ingreso a la UCI arrojó un VPN del $98 \%$ para descartar la mortalidad a los 28 días, mientras que un incremento del $9 \%$ en el día 7 arrojó un VPN del 97,2\%.

\section{Biomarcadores relacionados con la coagulación y la proteasa de neutrófilos}

Las proteasas de neutrófilos están involucradas en la patogénesis de las alteraciones de la coagulación observadas en la sepsis y median el daño endotelial. Hemos identificado que la actividad de la proteasa de neutrófilos (metalopeptidasa de matriz 8 y lipocalina-2-LCN2/NGAL) está asociada con el grado de falla orgánica y la mortalidad en la sepsis ${ }^{25}$. La evidencia de unos valores bajos o disminuidos de proteasas de neutrófilos durante la hospitalización es también una señal candidata adicional para identificar pacientes no complicados. Las proteasas de neutrófilos median la proteólisis, y usando espectrometría de masas Bauzá-Martínez, et al. ${ }^{26}$ demostraron que los sobrevivientes del shock séptico se caracterizan por la ausencia de una mayor proteólisis a lo largo de la evolución de la enfermedad (evidenciada por la abundancia de péptidos).

\section{Conclusiones}

Hay una serie de biomarcadores prometedores involucrados en alteraciones proteolíticas, vasculares, inmunitarias y de la coagulación que podrían ser útiles para construir endotipos compuestos que predicen resultados no complicados en la sepsis. Estos endotipos podrían ayudar a identificar a los pacientes que merecen el inicio temprano de antimicrobianos o la interrupción de estos. Se necesitan más esfuerzos con el fin de identificar las combinaciones óptimas de biomarcadores para obtener el endotipo mejor compuesto mediante evaluaciones repetidas a lo largo del curso de la enfermedad. Las nuevas tecnologías de metabolómica, las citometrías de flujo, los inmunoensayos y las tecnologías de cuantificación del ARN mensajero facilitarán la identificación precisa de estos endotipos.

\section{Financiamiento}

No se obtuvo ningún tipo de financiamiento por parte de ninguna empresa.

\section{Conflicto de intereses}

Los autores declaran que no existe ningún conflicto de intereses.

\section{Responsabilidades éticas}

Protección de personas y animales. Los autores declaran que para esta investigación no se han realizado experimentos en seres humanos ni en animales.

Confidencialidad de los datos. Los autores declaran que han seguido los protocolos de su centro de trabajo sobre la publicación de datos de pacientes.

Derecho a la privacidad y consentimiento informado. Los autores declaran que en este artículo no aparecen datos de pacientes.

\section{Bibliografía}

1. Wong HR, Cvijanovich N, Lin R, Allen GL, Thomas NJ, Willson DF, et al. Identification of pediatric septic shock subclasses based on genome-wide expression profiling. BMC Med. 2009;22:7-34.

2. Davenport EE, Burnham KL, Radhakrishnan J, Humburg P, Hutton P, Mills TC, et al. Genomic landscape of the individual host response and outcomes in sepsis: a prospective cohort study. Lancet Respir Med. 2016;4:259-71.

3. Burnham KL, Davenport EE, Radhakrishnan J, Humburg P, Gordon AC, Hutton $P$, et al. Shared and distinct aspects of the sepsis transcriptomic response to fecal peritonitis and pneumonia. Am J Respir Crit Care Med. 2017;196:328-39.

4. Scicluna BP, van Vught LA, Zwinderman AH, Wiewel MA, Davenport EE, Burnham KL, et al. Classification of patients with sepsis according to blood genomic endotype: a prospective cohort study. Lancet Respir Med. 2017;5:816-26.

5. Chuang CC, Wang ST, Chen WC, Chen CC, Hor LI, Chuang AY. Increases in serum macrophage migration inhibitory factor in patients with severe sepsis predict early mortality. Shock. 2007;27:503-6.

6. Monneret G, Finck ME, Vennet F, Debard AL, Bohé J, Bienvenu J, et al. The anti-inflammatory response dominates after septic shock: association of low monocyte HLA-DR expression and high IL-10 concentration. Immunol Lett. 2004;95:193-8.

7. Sweeney TE, Azad TD, Donato M, Haynes WA, Perumal TM, Henao R, et al. Unsupervised analysis of transcriptomics in bacterial sepsis across multiple datasets reveals three robust clusters. Crit Care Med. 2018;46:915-25

8. Kangelaris KN, Prakash A, Liu KD, Aouizerat B, Woodruff PG, Erle DJ, et al. Increased expression of neutrophil-related genes in patients with early sepsis-induced ARDS. Am J Phys Lung Cell Mol Phys. 2015;308:L1102-13.

9. Palmer C, Diehn M, Alizadeh AA, Brown PO. Cell-type specific gene expression profiles of leukocytes in human peripheral blood. BMC Genomics. 2006;7:115 
10. Spielman RS, Bastone LA, Burdick JT, Morley M, Ewens WJ, Cheung VG Common genetic variants account for differences in gene expression among ethnic groups. Nat Genet. 2007;39:226-31.

11. Hou PC, Filbin MR, Wang H, Ngo L, Huang DT, Aird WC, et al. Endothelial permeability and hemostasis in septic shock: results from the ProCESS trial. Chest. 2017;152:22-31.

12. van Vught LA, Wiewel MA, Hoogendijk AJ, Frencken JF, Scicluna BP Klein Klouwenberg PMC, et al. The host response in patients with sepsis developing intensive care unit-acquired secondary infections. Am J Respir Crit Care Med. 2017;196:458-70.

13. Wong HR, Salisbury S, Xiao Q, Cvijanovich NZ, Hall M, Allen GL, et al. The pediatric sepsis biomarker risk model. Crit Care. 2012;16:R174.

14. Wong HR, Cvijanovich NZ, Anas N, Allen GL, Thomas NJ, Bigham MT et al. Improved risk stratification in pediatric septic shock using both protein and mRNA biomarkers. PERSEVERE-XP. Am J Respir Crit Care Med. 2017;196:494-501.

15. Serkova NJ, Standiford TJ, Stringer KA. The emerging field of quantitative blood metabolomics for biomarker discovery in critical illnesses. Am J Respir Crit Care Med. 2011;184:647-55.

16. Eckerle $M$, Ambroggio $L$, Puskarich $M A$, Winston $B$, Jones $A E$, Standiford TJ, et al. Metabolomics as a driver in advancing precision medicine in sepsis. Pharmacotherapy. 2017;37:1023-32.

17. Mickiewicz B, Duggan GE, Winston BW, Doig C, Kubes P, Vogel HJ et al. Metabolic profiling of serum samples by $1 \mathrm{H}$ nuclear magnetic resonance spectroscopy as a potential diagnostic approach for septic shock. Crit Care Med. 2014;42:1140-9.

18. Ferrario M, Cambiaghi A, Brunelli L, Giordano S, Caironi P, Guatteri L, et al. Mortality prediction in patients with severe septic shock: a pilo study using a target metabolomics approach. Sci Rep. 2016;6:20391.
19. Rogers AJ, McGeachie M, Baron RM, Gazourian L, Haspel JA, Nakahira K, et al. Metabolomic derangements are associated with mortality in critically ill adult patients. PLoS One. 2014;9: e87538.

20. Langley RJ, Tsalik EL, van Velkinburgh JC, Glickman SW, Rice BJ, Wang $C$, et al. An integrated clinico-metabolomic model improves prediction of death in sepsis. Sci Transl Med. 2013;5:195ra195.

21. Neugebauer S, Giamarellos-Bourboulis EJ, Pelekanou A, Marioli A, Baziaka F, Tsangaris I, et al. Metabolite profiles in sepsis: developing prognostic tools based on the type of infection. Crit Care Med. 2016;44:1649-62.

22. Elke G, Bloos F, Wilson DC, Brunkhorst FM, Briegel J, Reinhart K, et al. The use of mid-regional proadrenomedullin to identify disease severity and treatment response to sepsis - a secondary analysis of a large randomised controlled trial. Crit Care. 2018;22:79.

23. Monneret G, Lepape A, Voirin N, Bohé J, Venet F, Debard A-L, et al. Persisting low monocyte human leukocyte antigen-DR expression predicts mortality in septic shock. Intensive Care Med. 2006;32:1175-83.

24. Wu J-F, Ma J, Chen J, Ou-Yang B, Chen M-Y, Li L-F, et al. Changes of monocyte human leukocyte antigen-DR expression as a reliable predictor of mortality in severe sepsis. Crit Care. 2011;15:R220.

25. Almansa R, Ortega A, Ávila-Alonso A, Heredia-Rodríguez M, Martín S, Benavides D, et al. Quantification of immune dysregulation by next-generation polymerase chain reaction to improve sepsis diagnosis in surgical patients. Ann Surg. 2019;269:545-53.

26. Bauzá-Martínez J, Aletti F, Pinto BB, Ribas V, Odena MA, Díaz R, et al. Proteolysis in septic shock patients: plasma peptidomic patterns are associated with mortality. Br J Anaesth. 2018;121:1065-74. 